\title{
Development of the Solar Neutron TRACking (SONTRAC) Concept
}

\author{
J. G. Mitchell, ${ }^{a, b, *}$ G. A. de Nolfo, ${ }^{b}$ A. Bruno, ${ }^{c, b}$ J. Dumonthier, ${ }^{b}$ I. Liceaga-Indart, ${ }^{b, c} \mathbf{J}$. \\ Link, ${ }^{d, b, \dagger}$ J. Legere, ${ }^{d}$ R. Messner, ${ }^{e}$ J. Ryan, ${ }^{d}$ G. Suarez ${ }^{b}$ and T. Tatoli ${ }^{c, b}$ \\ ${ }^{a}$ Department of Physics, George Washington University, Washington, DC, USA \\ ${ }^{b}$ NASA Goddard Space Flight Center, Greenbelt, MD, USA \\ ${ }^{c}$ Catholic University, Washington, DC, USA \\ ${ }^{d}$ University of Maryland, Baltimore County, Baltimore, MD, USA \\ ${ }^{d}$ Space Science Center, University of New Hampshire, Durham, NH, USA \\ ${ }^{e}$ ECE Department, College of Engineering and Physical Science, University of New Hampshire, Durham, \\ NH, USA \\ ${ }^{\dagger}$ No longer affiliated with NASA or CRESST as of May 2021 \\ E-mail: john.g.mitchell@nasa.gov
}

Fast neutrons ( $>0.5 \mathrm{MeV}$ ) are ubiquitous, originating from nuclear interactions in the solar corona, within planetary atmospheres, and in the lunar regolith. However, measurements of fast neutrons of solar origin are limited due to the challenges imposed by high backgrounds and the relatively short lifetime of free neutrons. Traditional double-scatter neutron spectrometers require an incident neutron to elastically scatter in two widely separated detectors, allowing the reconstruction of the incident neutron energy and direction. While double-scatter spectrometers are well-proven, they suffer from low effective area due to spacecraft size constraints and limited resolution. The Solar Neutron TRACking (SONTRAC) concept overcomes these limitations through the use of stacked planes of plastic scintillating fibers arranged in an orthogonal configuration, to measure the ionization tracks of recoil protons. The recoil proton energy and direction supplant the need to measure the neutron time-of-flight between detectors, thereby increasing the effective area and detection efficiency. SONTRAC employs modern, miniature silicon photomultipliers (SiPM) to measure the light output from the fibers. The SONTRAC concept, the development of a new fiber-bundle without an epoxy binder, testing of new high-performance applicationspecific-integrated-circuits, and development of new readout and reconstruction techniques are presented.

$37^{\text {th }}$ International Cosmic Ray Conference (ICRC 2021)

July 12th - 23rd, 2021

Online - Berlin, Germany

\footnotetext{
*Presenter
} 


\section{Introduction}

Magnetic reconnection processes within solar flares and jets efficiently accelerate charged particles. These charged particles, referred to as solar energetic particles (SEP), are guided along magnetic field lines in the low plasma $\beta$ solar corona. SEPs may escape along the open field lines into interplanetary space allowing in-situ measurement by instruments on spacecraft. However, SEPs can remain trapped along closed coronal loops and inaccessible to direct measurement. When these SEPs reach the base of the coronal loops on which they are trapped, they interact with the dense solar atmosphere and may produce neutrons and $\gamma$-rays. Because neutrons and $\gamma$-rays are electrically neutral, they can escape the coronal magnetic field and serve as probes to investigate the SEP acceleration mechanism as well as the nuclear interactions between solar flare SEPs and plasma in the solar atmosphere.

The spectrum of neutral radiation produced by the interaction of ions with footpoint plasma is governed by the energy spectrum and species of the accelerated ions and the composition of the ambient plasma. The dominant modes of photon radiation in a solare flare are bremsstrahlung X-ray emission from accelerated electrons often with a power-law spectrum, 1-10 MeV $\gamma$-rays produced by excited nuclei, and $>10 \mathrm{MeV} \gamma$-rays through the decay of secondary pions. In particular, the 1-10 MeV $\gamma$-ray lines produced by a combination of nuclear de-excitation, neutron capture, and positron annihilation can provide important information regarding both the ambient coronal plasma and the composition of particles accelerated by a solar flare [1].

Solar flare neutrons are produced via nuclear spallation interactions, predominantly from $\mathrm{p}-\mathrm{p}$, $\mathrm{p}-\alpha$, and $\alpha-\alpha$ interactions. Examination of the characteristics of solar photon and neutron events indicate that these interactions occur in a region with ambient proton density between $10^{12}$ and $10^{16} \mathrm{~cm}^{-3}$, i.e., the solar chromosphere and photosphere [2,3]. As shown in Fig. 1a, fast neutrons between 20 and $500 \mathrm{MeV}$ bridge the gap between the low-energy $\gamma$-rays produced by nuclear deexcitation and the high-energy pion-produced $\gamma$-rays [4, 5]. Thus, solar neutron measurements are critical for characterizing the full secondary neutral spectrum produced by flare-accelerated ions, and the spectrum of the ions themselves.

The spectral and compositional information, and the combined measurement of solar neutrons and $\gamma$-rays provide important temporal information regarding the evolution of a flare. For example, in the case of the 1991 June 9 solar flare, the COMPTEL Imaging Compton Telescope on CGRO observed the flare neutrons as well as the low and high-energy $\gamma$-rays and found that the nuclear line emission $\gamma$-rays peaked first, followed by the neutrons, and finally the high-energy pion-produced $\gamma$-rays as shown in Fig. 1b [5, 6]. This indicates that the accelerated proton spectrum evolved throughout the solar flare, beginning with a fairly typical proton spectrum but hardening as the flare progressed.

Free neutrons are challenging to measure in part due to the $887 \mathrm{~s}$ lifetime. Thus, measurements at $1 \mathrm{AU}$ are effectively restricted to energies above $10 \mathrm{MeV}$. The survival probability for neutrons of various energies is shown as a function of heliocentric distance in Fig. 2. This illustrates the challenge of detecting solar neutrons at $1 \mathrm{AU}$ due to the $<50 \%$ survival probability of $<200$ $\mathrm{MeV}$ protons. With less than fifteen solar neutron events observed, and all at $1 \mathrm{AU}$, a composite measurement at small heliocentric distances of solar neutrons and $\gamma$-rays is a critical missing piece in quantifying the charged particle spectrum in solar flares. 

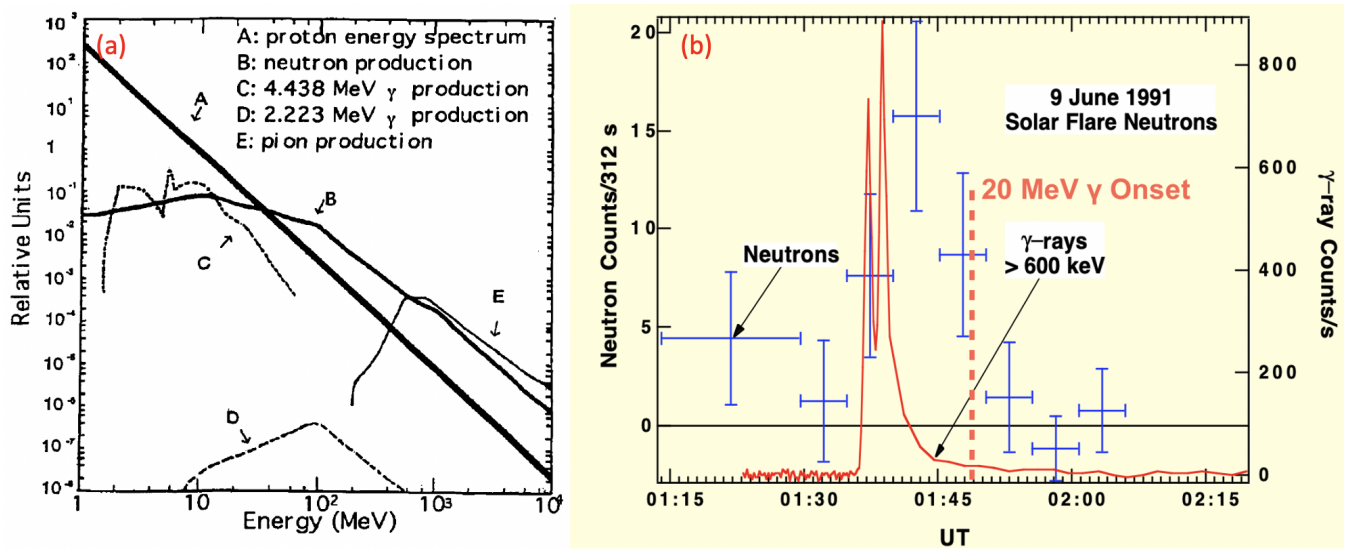

Figure 1: (a) Interacting ion spectrum (from [4, 5]). Solar neutrons (B) bridge the energy gap between the low-energy $\gamma$-rays produced by nuclear de-excitation (C) and high-energy pion-produced $\gamma$-rays (E). (b) Time-evolution of solar neutrons and $\gamma$-rays measured by COMPTEL on CGRO during the 1991 June 9 solar flare (adaped from [6])

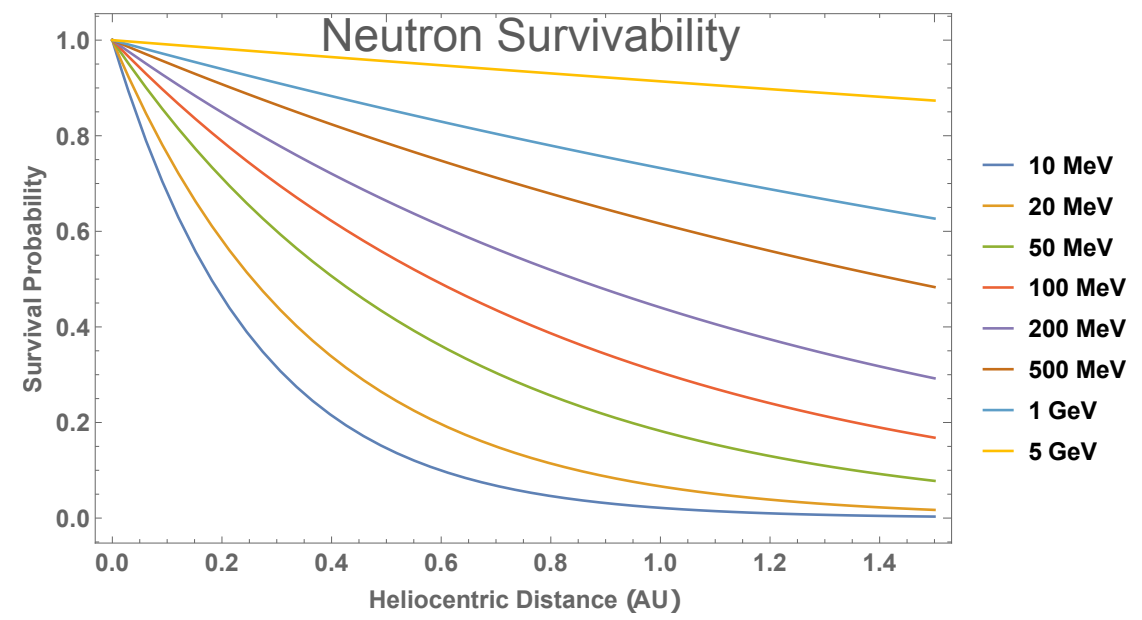

Figure 2: Survival probability of neutrons for several energies as a function of heliocentric distance.

\section{SONTRAC Concept and Design}

Traditional neutron spectrometers employ the double-scatter technique in which a neutron scatters in two detectors spaced relatively far apart (e.g. [7, 8]). With the double-scatter technique, one can reconstruct the energy of the incident neutron using the energy of the first recoil proton (obtained using the pulse height in the first detector hit) and the scattered neutron time-of-flight (ToF) between detectors. The neutron's incident direction can also be calculated to lie on the surface of a cone based on non-relativistic n-p elastic scattering kinematics. The half-angle of the cone, $\phi$, is given by $\sin ^{2} \phi=E_{p 1} / E_{n 0}$ where $E_{p 1}$ is the energy of the recoil proton in the first detector hit and $E_{n 0}$ is the energy of the incident neutron. As a consequence, direct imaging of the neutron source is not possible using a double-scatter spectrometer. While double-scatter neutron spectrometers have demonstrated their capability in measuring neutron energy, these instruments have a low effective area due to the requirement that the two detectors be spaced relatively far apart, resulting in a large 
amount of empty space between the detectors. Thus, double-scatter neutron spectrometers require large spacecraft and are challenging to fly on modern compact spacecraft (e.g. CubeSats or probes). Additionally, in the case of incident neutron energies $\gtrsim 50 \mathrm{MeV}$, the recoil proton has a relatively high probability of leaving the detector, yielding an incomplete energy measurement.

To overcome the short-comings of traditional double-scatter neutron spectrometers, the Solar Neutron TRACking (SONTRAC) instrument utilizes a single large-volume detector composed of stacked orthogonal planes of scintillating plastic fibers [9-11]. With this detector construction, both neutron scatters may occur within a single detector volume and the detailed path of the recoil protons can be measured. Well-understood non-relativistic n-p elastic scattering kinematics allow the reconstruction of the incident neutron's energy and direction based on the detailed energy and momentum vectors of the recoil protons as shown in Fig. 3a. As shown in Fig. 3b, the fact that the SONTRAC fiber bundle is a single large volume means there is significantly less insensitive volume than in a traditional double-scatter neutron spectrometer resulting in a much larger effective area and the ability to efficiently measure solar neutrons with a much smaller instrument. With this type of detector, the ToF measurement is replaced by the recoil proton momentum vectors and the energy of the recoil proton can be determined based on the proton's track length through the bundle. Fig. 3c shows a scatter plot of recoil proton energy vs. track length from Geant4 [12] simulations of the SONTRAC fiber bundle (divided into the recoil proton scattered in the first neutron interaction and the second interaction) with the theory energy loss curve. The spread in the points around the theory line is due to the uncertainty of the exact position within a fiber that the n-p interaction occurred as well as the uncertainty in the precise end of the proton track. This uncertainty has a greater effect for shorter tracks than long tracks.

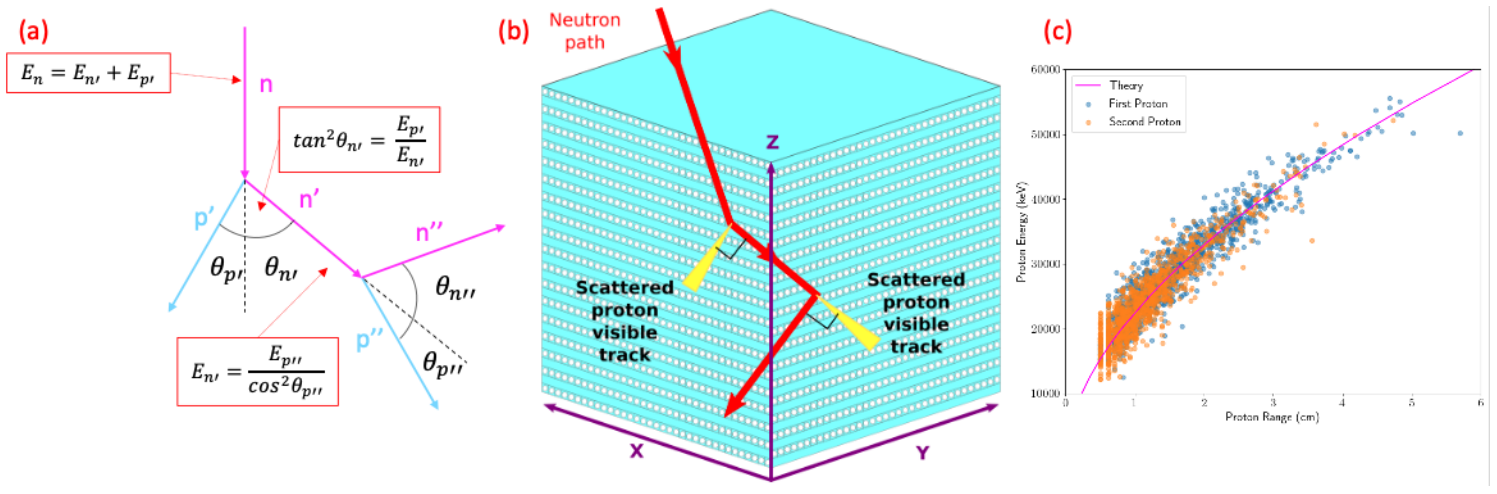

Figure 3: (a) Non-relativistic n-p elastic scattering kinematics for two neutron scatters. Equations show how one can reconstruct the energy and direction of the incident neutron based on the two measured recoil proton vectors. (b) Schematic of double-scatter event within the SONTRAC fiber bundle. Neutron path is shown by red arrows. Yellow triangles represent recoil proton tracks. (c) Proton energy vs. track length from Geant4 simulations of SONTRAC fiber bundle showing that the recoil proton energy can be determined by the track length through the fiber bundle.

\section{Recent SONTRAC Progress}

As described in de Nolfo et al., 2019 [11], the current SONTRAC fiber bundle design consists of planes of plastic scintillating fibers of $1.36-\mathrm{mm}$ pitch organized into a $\sim 10 \times 10 \times 10 \mathrm{~cm}^{3}$ cube as 
shown in Fig. 4a. Previous iterations of the SONTRAC fiber bundle utilized a black epoxy binder between the fibers to provide mechanical support as well as light isolation. This epoxy binder adds an enormous amount of passive material in which neutrons may scatter and recoil protons may lose energy that cannot be measured. This introduces significant uncertainty when trying to measure the detailed recoil proton energy and momentum vectors. Recently, a new fiber bundle was developed in which the fibers are fixed to a mechanical frame at each end supplanting the need to embed the fibers in epoxy as shown in Fig. 4b. This new fiber bundle allows recoil protons to travel through more fibers resulting in both longer proton tracks and more accurate measurements of the proton energy deposit, thereby improving reconstruction efficiency and accuracy and lowering the effective detection threshold energy.

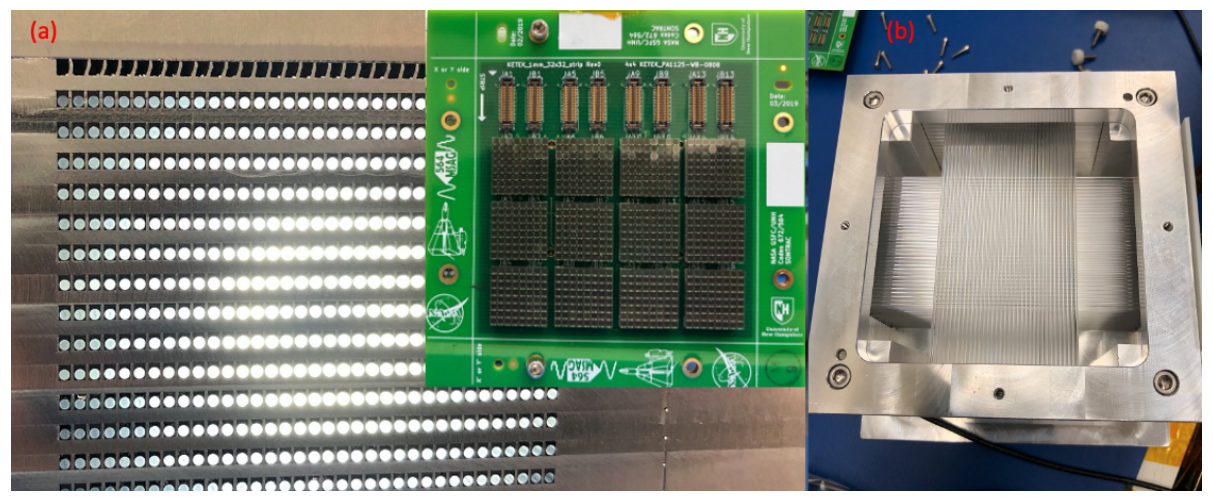

Figure 4: (a) Photograph of one side of current SONTRAC fiber bundle showing 1-mm plastic scintillating fibers organized into planes. Inset shows twelve $8 \times 8$ arrays of 1-mm SiPMs on a custom FEE board. (b) Top-down view of new SONTRAC fiber bundle without epoxy binder.

The light from the SONTRAC fibers is read by commercially available $8 \times 8$ arrays of finegrained 1-mm silicon photomultipliers (SiPM) by KETEK as shown in the inset of Fig. 4a. SiPMs are a type of solid-state photodetector that has benefitted from rapid technological advances in recent years. SiPMs are used in place of traditional photomultiplier tubes (PMT) and offer a substantial reduction in size, mass, and bias voltage compared with PMTs. In addition, SiPM characteristics include sub-ns rise times, low noise, and good quantum efficiency, making them an ideal photodetector for a compact instrument. The SiPMs are connected to custom front-endelectronics (FEE) boards designed by members of the SONTRAC team (see green PCB board in the inset of Fig. 4a). Several different configurations of FEE boards have been designed to read either single SiPM anodes or to sum groups of SiPMs (described in greater detail below).

Recently, members of the SONTRAC team have been investigating two commercial readout application specific integrated circuits (ASIC), the CAEN Petiroc $2 \mathrm{~A}^{1}$ and the PETsys TOFPET2 ${ }^{2}$, to process signals from the SiPMs. Both ASICs provide good time resolution, a high number of readout channels, and flexibility to customize the desired readout configuration. Investigations into the timing characteristics, triggering options, dynamic range, and energy resolution are in progress and will be used to optimize the SONTRAC readout electronics configuration. For example, recently laboratory tests with a $3 \times 3 \times 5 \mathrm{~mm}^{3}$ LYSO crystal have demonstrated that the SONTRAC SiPM

${ }^{1} \mathrm{https}: / / \mathrm{www} . c a e n . i t /$ products/dt5550w/

${ }^{2} \mathrm{https} / / / \mathrm{www}$.petsyselectronics.com/web/public/products/2 
arrays readout by the CAEN Petiroc2A ASIC can distinguish the intrinisic structure of the LYSO spectrum (Fig. 5a). The structure of the intrinisic LYSO spectrum is due to the contribution of an 88,202 , and $307 \mathrm{keV} \gamma$-ray decay product as well as combinations of the three [13]. As shown in Alva-Sánchez et al., 2018, the relative contribution of each of the $\gamma$-rays in LYSO depends on the size of the crystal. Geant 4 simulations of a $3 \times 3 \times 5 \mathrm{~mm}^{3}$ LYSO crystal (Fig. 5b) show that the higher peak in Fig. 5a is due to the combination of the $88 \mathrm{keV}$ and $202 \mathrm{keV} \gamma$-rays and the lower-energy shoulder is a result of individual $88 \mathrm{keV}$ and $202 \mathrm{keV} \gamma$-rays, demonstrating that this readout configuration is sensitive to $<200 \mathrm{keV} \gamma$-rays. The data shown in Fig. 5a was taken with two different SiPMs coupled to the same LYSO crystal but with the crystal coupled closely to one SiPM (shown in white) and with a small air gap between the crystal and the other SiPM (shown in purple). While the features remain at approximately the same ADC location, demonstrating equal gain, the relative intensity of the combination of $\gamma$-rays is effected by this difference in coupling. A method of consistent coupling is currently being devised such that a gain calibration and ADC-energy conversion for the SONTRAC readout electronics may be performed using LYSO.
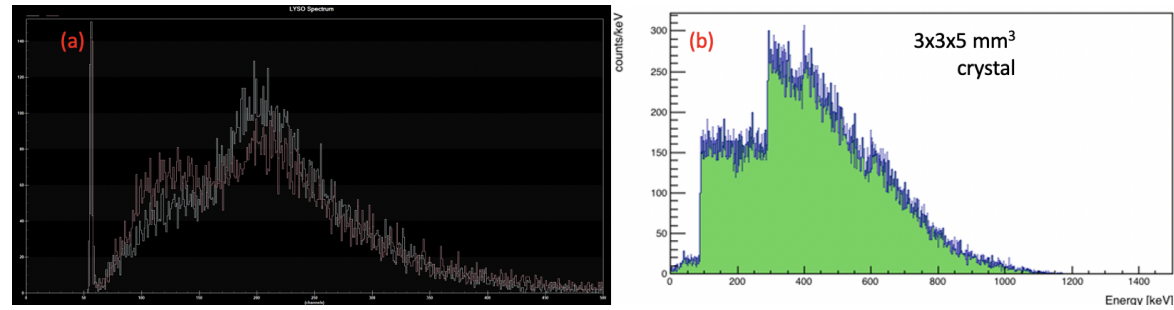

Figure 5: (a) Intrinisic spectrum of a $3 \times 3 \times 5 \mathrm{~mm}^{3}$ LYSO crystal as measured by a SONTRAC 1-mm SiPM array on a custom FEE board processed by the CAEN Petiroc 2A ASIC. The two spectra are from 2 individual SiPMs with slightly different optical coupling to the LYSO crystal. This results in differences in the relative detection efficiency of the combinations of $\gamma$-ray signals, but no appreciable difference in the relative gain between the channels. (b) Geant4 simulations of the intrinisic spectrum of a $3 \times 3 \times 5 \mathrm{~mm}^{3}$ LYSO crystal.

To read the signal from each individual SiPM on SONTRAC would require nearly 2500 individual readout channels. While this could be accomplished in the laboratory, reading out this number of channels in flight is unrealistic. To reduce the required number of readout channels, SONTRAC has used a configuration in which SiPMs are summed using custom FEE boards along $1-\mathrm{d}$ strips. With this technique, all four sides of the bundle are read and analyzed as "X", "Y", "XZ", and "YZ", respectively. This results in a significant reduction in the required number of channels to $\sim 100$. Track reconstruction is performed using sparse back-projection techniques used extensively in positron emission tomography (PET) imaging (e.g. [14]). An example of the track of a ground-level cosmic ray muon through the fiber bundle reconstructed using this technique is shown in Fig. 6a. Reconstruction via back-projection of the 1-d strip data has proven to be very effective, but can result in ambiguous tracks or tracks that fail to successfully reconstruct due to a lack of information. To improve the track reconstruction efficiency, a new summing technique in which non-adjacent groups of SiPMs are electronically summed using new custom FEE boards has been developed. This new coded summing technique, which is similar to the mechanical summing employed by the TIGER collaboration $[15,16]$, uses a "coarse" summing on one side in which adjacent groups of 8 SiPMs are summed and a "fine" summing on the opposite side in which SiPMs 
are summed sequentially based on their position within a coarse group as shown in Fig. 6b. This technique will dramatically improve the SONTRAC reconstruction efficiency and retrieve events that would be ambiguous using the 1-d strip summing technique.
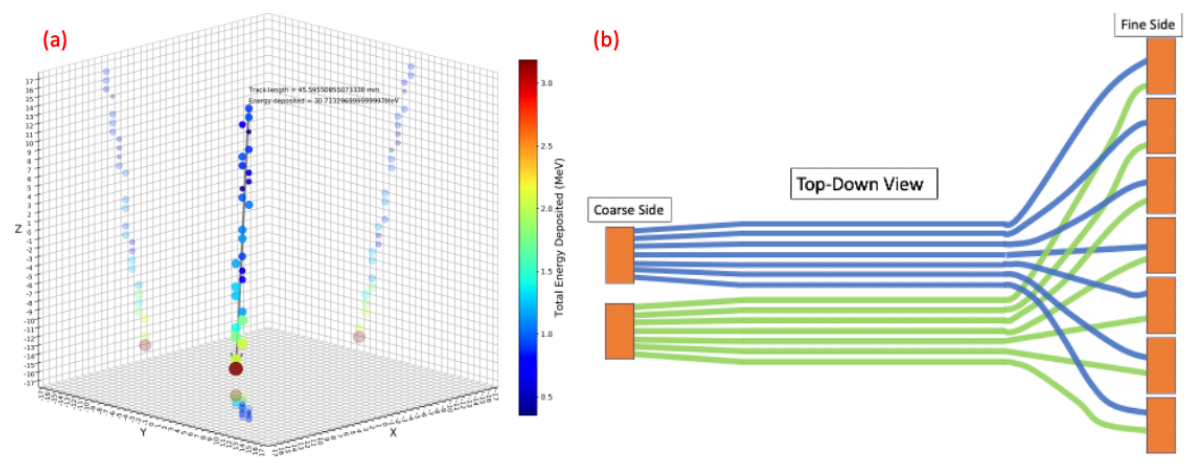

Figure 6: (a) Ground-level cosmic ray muon track through SONTRAC fiber bundle. This measurement was taken using summed 1-d strips of SiPMs and reconstructed using sparse back-projection techniques. (b) Illustration of the coded-readout scheme in which SiPMs are represented by orange boxes and the blue and green lines represent two different groups of fibers. The summing is done electronically, but this figure shows the concept done by mechanically routing fibers to illustrate the concept.

SONTRAC will be tested with mono-energetic protons and neutrons in an accelerator beam in Fall 2021 at Crocker Nuclear Laboratory at University of California Davis. The full SONTRAC instrument including fiber bundle and readout electronics will be characterized using data from this beam test. Once fully characterized, SONTRAC will be an ideal compact neutron spectrometer for a variety of space-based applications including CubeSats, solar or terrestrial probes, and lunar landers. SONTRAC can provide significant scientific value in a variety of applications.

\section{References}

[1] Murphy, R. J., R. Ramaty, and B. Kozlovsky. "Solar abundances from gamma-ray spectroscopy: Comparisons with energetic particle, photospheric, and coronal abundances." In AIP Conference Proceedings, vol. 232, no. 1, pp. 439-444. American Institute of Physics, 1991.

[2] Chupp, E. L. "High-energy neutral radiations from the sun." Annual review of astronomy and astrophysics 22 , no. 1 (1984): 359-387.

[3] Hua, X-M., and R. E. Lingenfelter. "Solar flare neutron production and the angular dependence of the capture gamma-ray emission." Solar Physics 107, no. 2 (1987): 351-383.

[4] Lockwood, J. A., H. Debrunner, and J. M. Ryan. "The relationship between solar flare gammaray emission and neutron production." Solar Physics 173, no. 1 (1997): 151-176.

[5] Ryan, James M., Laurent Desorgher, Erwin O. Flueckiger, John R. Macri, Mark L. McConnell, and Richard S. Miller. "SONTRAC: An imaging spectrometer for solar neutrons." In Innovative Telescopes and Instrumentation for Solar Astrophysics, vol. 4853, pp. 399-410. International Society for Optics and Photonics, 2003. 
[6] Ryan, J., K. Bennett, H. Debrunner, D. Forrest, L. Hanlon, J. Lockwood, M. Loomis et al. "Neutron and gamma ray measurements of the solar flare of 1991 June 9." In 23rd International Cosmic Ray Conference (ICRC23), Volume 3, vol. 3, p. 103. 1993.

[7] Ryan, James, K. Bennett, H. Debrunner, D. Forrest, J. Lockwood, M. Loomis, M. McConnell et al. "Comptel measurements of solar flare neutrons." Advances in Space Research 13, no. 9 (1993): 255-258.

[8] Mitchell, J. G., A. Bruno, S. Candey, G. A. de Nolfo, J. Dumonthier, I. Liceaga-Indart, I. Rankin, K. Rankin, J. M. Ryan, S. Stochaj, and G. Suarez. "Performance Characteristics of the Ionospheric Neutron Content Analyzer (INCA)." In 36th International Cosmic Ray Conference (ICRC2019), vol. 36, p. 53. 2019.

[9] Frye Jr, G. M., T. L. Jenkins, and A. Owens. "SONTRAC: A solar neutron track chamber detector." In 19th International Cosmic Ray Conference (ICRC19), Volume 5, vol. 5. 1985.

[10] Miller, Richard S., John R. Macri, Mark L. McConnell, James M. Ryan, Erwin Flückiger, and Laurent Desorgher. "SONTRAC: An imaging spectrometer for MeV neutrons." Nuclear Instruments and Methods in Physics Research Section A: Accelerators, Spectrometers, Detectors and Associated Equipment 505, no. 1-2 (2003): 36-40.

[11] De Nolfo, G. A., A. Bruno, J. Dumonthier, I. Liceaga-Indart, J. Legere, R. Messner, J. G. Mitchell, J. M. Ryan, G. Suarez, and T. Tatoli. "SOlar Neutron TRACking (SONTRAC) Concept." In 36th International Cosmic Ray Conference (ICRC2019), vol. 36, p. 1074. 2019.

[12] Agostinelli, Sea, John Allison, K. al Amako, John Apostolakis, H. Araujo, P. Arce, M. Asai et al. "GEANT4 - a simulation toolkit." Nuclear instruments and methods in physics research section A: Accelerators, Spectrometers, Detectors and Associated Equipment 506, no. 3 (2003): 250-303.

[13] Alva-Sánchez, H., A. Zepeda-Barrios, V. D. Díaz-Martínez, T. Murrieta-Rodríguez, A. Martínez-Dávalos, and M. Rodríguez-Villafuerte. "Understanding the intrinsic radioactivity energy spectrum from $176 \mathrm{Lu}$ in LYSO/LSO scintillation crystals." Scientific reports 8, no. 1 (2018): 1-7.

[14] Kak, Avinash C., and Malcolm Slaney. "Principles of computerized tomographic imaging. The Institute of Electrical and Electronics Engineers." Inc., New York (1988).

[15] Lawrence, D. J., L. M. Barbier, J. J. Beatty, W. R. Binns, E. R. Christian, D. J. Crary, D. J. Ficenec et al. "Large-area scintillating-fiber time-of-flight/hodoscope detectors for particle astrophysics experiments." Nuclear Instruments and Methods in Physics Research Section A: Accelerators, Spectrometers, Detectors and Associated Equipment 420, no. 3 (1999): 402-415.

[16] Ward, J. E., W. R. Binns, R. G. Bose, T. J. Brandt, D. L. Braun, W. M. Daniels, G. A. De Nolfo et al. "The Super-TIGER Scintillating Fiber Hodoscope." In International Cosmic Ray Conference, vol. 33, p. 2136. 2013. 